[7] T. Popoviciu, Sur l'approximation de functions convexes d'ordre superieur, Mathematica, 10, 49 to 54 (1935).

[8] E. Voronovskaja, Détermination de la forme asymptotique de l'approximation des fonctions par les polynômes de S. Bernstein, Compt. Rend. l' acad. sci. URSS, 4, 79 to 85 (1932).

Attention is called to relevant work of Einar Hille (see

Proc. Nat. Acad. Sci., p. 421 to 424 (Oct. 28, 1942), and his book: Functional analysis and semi-groups, Am. Math. Soc. Colloqu. publications 31, 186 to 189 , 323 to 325,332 to 333 (1948), where transform (2) is dealt with as a special case of a more general transformation. It contains in particular some part of the results of this paper.

Los Angeles, November 9, 1949.

\title{
Numerical Determination of Characteristic Numbers ${ }^{1}$
}

\author{
By W. E. Milne
}

\begin{abstract}
This paper represents a contribution to the problem of characteristic values and characteristic solutions of ordinary linear differential equations. The problem is conceived as a vibration problem in $x$ and $t$. The partial differential equation is then approximated by a difference equation in both entries. The problem is now to find those frequencies in which a separation in $x$ and $t$ takes place. This is done by finding the roots of a trigonometric expansion of certain order. The method is applied to a number of interesting cases that illustrate various types of situations encountered in problems of physics and engineering.
\end{abstract}

An improved method for obtaining the latent roots of a matrix has been devised by Cornelius Lanczos of this Bureau. He has shown further how this process may be used to secure the characteristic numbers (eigenwerte) belonging to a boundary value problem associated with an ordinary linear differential equation. The purpose of this note is to present a procedure for calculating characteristic numbers, based essentially on this method of Lanczos, but modified in such a way as to provide a simple numerical routine for the computation. A number of numerical examples, worked in full, illustrate the procedure and give an indication of the accuracy attained. The exposition is limited to the case of differential equations of the second order, but the method is capable of extension to cases of higher order.

1. The problem. Let

$$
L(u)=P_{0}(x) \frac{d^{2} u}{d x^{2}}+P_{1}(x) \frac{d u}{d x}+P_{2}(x) u .
$$

The problem before us is to find those characteristic values of $\lambda$ for which the differential system

$$
\begin{aligned}
& L(u)+\lambda^{2} u==0 \\
& \left.\begin{array}{l}
u+g \frac{d u}{d x}=0 \text { at } x=a \\
u+G \frac{d u}{d x}=0 \text { at } x=b
\end{array}\right\}
\end{aligned}
$$

possesses nonzero solutions in the interval $a \leqq x \leqq b$.

Problems of this type arise in many different ways in mathematical physics. For example, they may occur in connection with the heat equation

$$
\frac{\partial V}{\partial t}=L(V)
$$

if we assume particular solutions of the form

$$
V=u(x) e^{-\lambda^{2} t},
$$

or in connection with the wave equation

$$
\frac{\partial^{2} V}{\partial t^{2}}=L(V)
$$

1 The preparation of this paper was sponsored (in part) by the Office of Naval Research. 
if we assume particular solutions of the form

$$
V=u(x) \cos \lambda t,
$$

or they may occur after separation of variables in more general partial differential equations.

The important fact is that the characteristic numbers are completely determined by the system 1, regardless of the particular physical problems from which 1 may have been derived. Consequently, in order to obtain numerical solution we may assume a fictitious partial differential system which leads to (1). The most satisfactory fictitious system for our purpose appears to be

$$
\frac{\partial^{2} V}{\partial t^{2}}=L(V)
$$

with boundary conditions

$$
\left.\begin{array}{l}
V+g \frac{\partial V}{\partial x}=0 \text { at } x=a, \\
V+G \frac{\partial V}{\partial x}=0 \text { at } x=b,
\end{array}\right\}
$$

and initial conditions

$$
\left.\begin{array}{c}
V=F(x) \text { when } t=0, \\
\frac{\partial V}{\partial t}=0 \text { when } t=0 .
\end{array}\right\}
$$

Following standard procedure, we assume that the solution of $(2,3$, and 4$)$ is

$$
V=\sum_{k} c_{k} u_{k}(x) \cos \lambda_{k} t
$$

where the $\lambda_{k}$ are the desired characteristic numbers, the $u_{k}(x)$ are the characteristic functions (normalized in some definite manner) and the $c_{k}$ are constants depending on $F(x)$.

2. Normalization of $L(u)$. It will be assumed with respect to the coefficient functions in $L(u)$ that for all values of $x$ in the interval $a \leqq x \leqq b$

$$
\text { (a) } P_{0}(x)>0 \text {, }
$$

(b) $\frac{d}{d x} P_{0}(x)$ exists and is continuous,

(c) $P_{1}(x)$ and $P_{2}(x)$ are continuous.

We find it desirable to change the independent variable $x$ to a new variable $s$ by the relation
This change of variable carries $L(u)$ into what we shall call the "normal form"

$$
L(u)=\frac{d^{2} u}{d s^{2}}+p(s) \frac{d u}{d s}+q(s) u,
$$

where $p(s)$ and $q(s)$ are seen to be continuous in the interval $a^{\prime} \leqq s \leqq b^{\prime}$ corresponding to $a \leqq x \leqq b$. It will be assumed from now on that $L(u)$ has already been put in the normal form.

3. The partial difference equation. Let the interval $a \leqq x \leqq b$ be divided into $n+1$ equal subintervals of length

$$
h=\frac{b-a}{n+1},
$$

and let the value of $V(x, t)$ at the point $x=a+i h$ and at time $t=j h$, be designated by $V_{i j}$. Then in the differential equation

$$
\frac{\partial^{2} V}{\partial t^{2}}=\frac{\partial^{2} V}{\partial x^{2}}+p(x) \frac{\partial V}{\partial x}+q(x) V
$$

we make the substitutions

$$
\begin{aligned}
& \frac{\partial^{2} V}{\partial t^{2}}=\frac{V_{i, j+1}-2 V_{i j}+V_{i, j-1}}{h^{2}}, \\
& \frac{\partial^{2} V}{\partial^{2} x}=\frac{V_{i+1, j}-2 V_{i j}+V_{i-1, j}}{h^{2}}, \\
& \frac{\partial V}{\partial x}=\frac{V_{i+1, j}-V_{i-1, j}}{2 h},
\end{aligned}
$$

and after simplification obtain the approximate difference equation

$$
\begin{aligned}
V_{i, j+1}+V_{i, j-1} & =V_{i+1, j}+V_{i-1, j} \\
& +\frac{h p_{i}}{2}\left[V_{i+1, j}-V_{i-1, j}\right]+h^{2} q_{i} V_{i j},
\end{aligned}
$$

in which

$$
p_{i}=p(a+i h), \quad q_{i}=q(a+i h) .
$$

If we assume the existence and continuity of the second derivatives of $p(x)$ and $q(x)$, and suppose that the error of eq 6 is expanded in powers of $h$, we find that the leading term of the error is

$$
\begin{gathered}
\frac{h^{4}}{12}\left[\left(p^{2}+2 p^{\prime}+2 q\right)\right. \\
\frac{\partial^{2} V}{\partial x^{2}}+\left(p^{\prime \prime}+p p^{\prime}+2 q^{\prime}+2 p q\right) \frac{\partial V}{\partial x} \\
\left.+\left(q^{\prime \prime}+p q^{\prime}+q^{2}\right) V\right],
\end{gathered}
$$


showing that the error of eq 6 is of fourth order in $h$.

4. Derivation of the characteristic equation. For simplicity in the following explanation, let us suppose that in the boundary conditions $g=G=0$, so that $V_{o j}=0, V_{n+1, j}=0$. For $t=0$ (or $j=0$ ) the values of $V_{i 0}$ are arbitrary (being equal to the arbitrary function $F(x)$ ). Hence we choose these in the most convenient manner, say $V_{10} \neq 0$, $V_{i 0}=0, i>1$. Then we have for $V_{i j}$ the array of values

\begin{tabular}{|c|c|c|c|c|c|c|c|}
\hline$i$ & 0 & 1 & 2 & 3 & $\cdots$ & $n$ & $n+1$ \\
\hline 0 & 0 & $V_{10}$ & 0 & 0 & $\ldots$ & 0 & 0 \\
\hline 1 & 0 & $V_{11}$ & $V_{21}$ & 0 & & 0 & 0 \\
\hline 2 & 0 & $V_{12}$ & $V_{22}$ & $V_{32}$ & & 0 & 0 \\
\hline 3 & 0 & $V_{13}$ & $V_{23}$ & $V_{33}$ & & 0 & 0 \\
\hline . . & . . & . . & . . & . . & $\cdot \cdot$ & . . . & . . \\
\hline$n-1$ & 0 & $V_{1 n-1}$ & $V_{2 n-1}$ & $V_{3 n-1}$ & & $V_{n n-1}$ & 0 \\
\hline$n$ & 0 & $V_{2 n}$ & $V_{1 n}$ & $V_{3 n}$ & & $V_{n n}$ & 0 \\
\hline
\end{tabular}

As just explained, the entries of the first row are arbitrary and are chosen as shown for convenience. To obtain the second row we use eq 6 , together with the relation

$$
V_{i, 1}=V_{i,-1},
$$

derived from the condition $\partial V / \partial t=0$ at $t=0$. All remaining rows are computed by eq 6 .

So far no restriction has been placed on the magnitude of the interval $h$. We now impose the condition that $h$ is chosen small enough that

$$
\left|\frac{1}{2} h p_{i}\right|<1,
$$

for all values of $i$.

It will then be seen from array 8, together with eq 6 , that the entry in the principal diagonal in the second column is given by

$$
V_{21}=\frac{1}{2}\left(1-\frac{1}{2} h p_{2}\right) V_{10},
$$

whereas for subsequent columns the entries in the principal diagonal are

$$
\begin{aligned}
& V_{32}=\left(1-\frac{1}{2} h p_{3}\right) V_{21}, \\
& V_{43}=\left(1-\frac{1}{2} h p_{4}\right) \cdot V_{32},
\end{aligned}
$$

etc.

Since by our choice $V_{10} \neq 0$ no entry in the principal diagonal is zero, and the $n$-square matrix obtained from (8) by dropping the first and last columns and the last row is not singular. Then there will exist $n$ constants $A_{0}, A_{1}, \ldots, A_{n-1}$ such that

$$
\sum_{j=0}^{n} A_{j} V_{i j}=0 \text { for } i=1,2, \ldots, n,
$$

(where $A_{n}=1$ ).

Now from eq 5 we have

$$
V_{i j}=\sum_{k=1}^{n} c_{k} u_{k i} \cos \lambda_{k} h j,
$$

where $u_{k i}=u_{k}(a+i h)$. Therefore,

$$
\sum_{k=1}^{n} c_{k} u_{k i} \sum_{j=0}^{n} A_{j} \cos \lambda_{k} h j=0, \quad i=1,2, \ldots, n .
$$

It can be shown that these equations imply

$$
\sum_{j=0}^{n} A_{j} \cos \lambda_{k} h j=0, \quad k=1,2, \ldots, n .
$$

It is apparent, therefore, that the desired characteristic numbers $\lambda_{k}$ are given by

$$
\lambda_{k}=\mu_{k} / h, \quad k=1,2, \ldots, n,
$$

where $\mu_{1}, \mu_{2}, \ldots, \mu_{n}$ are roots of the characteristic equation

$\cos n \mu+A_{n-1} \cos (n-1) \mu+\cdots+A_{1} \cos \mu+A_{0}=0$.

The $\lambda_{k}$ thus found are correct for the difference equation with the given boundary conditions but in general are only approximations to the $\lambda_{k}$ belonging to the differential system 1 .

5. The characteristic functions. In order to obtain numerical values for the characteristic function $u_{k i}$ associated with $\lambda_{k}$, we substitute

in eq 6 . Since

$$
V_{i j}=u_{k i} \cos \mu_{k} j
$$

$$
V_{i, j+1}+V_{i, j-1}=2 V_{i j} \cos \mu_{k},
$$

the result, after removal of the factor $\cos \mu_{k} j$, may be put in the form

$$
\begin{gathered}
\left(1+\frac{1}{2} h p_{i}\right) u_{k, i+1}=\left(2 \cos \mu_{k}-h^{2} q_{i}\right) u_{k i}- \\
\left(1-\frac{1}{2} h p_{i}\right) u_{k, i-1} .
\end{gathered}
$$

This is an ordinary difference equation of second order. We may choose any nonzero value for $u_{k, 1}$, say

$$
u_{k 1}=1 \text {. }
$$


Since $u_{k 0}=0$ we obtain $u_{k, 2}$ from eq 10 , then $u_{k, 3}$, etc. The fact that $u_{k, n+1}$ should turn out to be zero serves as a check on the calculations.

As a very simple example, let us take the system

$$
\begin{gathered}
\frac{d^{2} u}{d x^{2}}+\lambda^{2} u=0, \\
u=0 \quad \text { at } x=0, \\
u=0 \quad \text { at } x=1 .
\end{gathered}
$$

Let $h=1 / 6$. For this system eq 6 becomes

$$
V_{i, j+1}+V_{i, j-1}=V_{i+1, j}+V_{i-1, i},
$$

and if we take $V_{1,0}=2$ the array (8) turns out to be

$\begin{array}{rrrrrrr}0 & 2 & 0 & 0 & 0 & 0 & 0 \\ 0 & 0 & 1 & 0 & 0 & 0 & 0 \\ 0 & -1 & 0 & 1 & 0 & 0 & 0 \\ 0 & 0 & -1 & 0 & 1 & 0 & 0 \\ 0 & 0 & 0 & -1 & 0 & 1 & 0 \\ 0 & 0 & 0 & 0 & -1 & 0 & 0\end{array}$

From this we have inmediately

$$
\begin{aligned}
& A_{0}=A_{2}=A_{4}=0, \\
& A_{1}=A_{3}=1,
\end{aligned}
$$

and the characteristic equation is

$$
\cos 5 \mu+\cos 3 \mu+\cos \mu=0,
$$

or in factored form

$$
\cos 3 \mu(2 \cos \mu+1)=0 .
$$

From the second form we read off the roots at once.

whence

$$
\mu_{1}=\frac{\pi}{6}, \mu_{2}=\frac{2 \pi}{6}, \mu_{3}=\frac{3 \pi}{6}, \mu_{4}=\frac{4 \pi}{6}, \mu_{5}=\frac{5 \pi}{6},
$$

$$
\lambda_{k}=k \pi, \quad k=1,2,3,4,5 .
$$

To obtain values of the characteristic functions, we resort to eq 10 , which here becomes

$$
u_{k, i+1}=2\left(\cos \mu_{k}\right) u_{k, i}-u_{k, i-1},
$$

where $\cos \mu_{1}=\frac{1}{2} \sqrt{3}, \cos \mu_{2}=\frac{1}{2}, \cos \mu_{3}=0, \cos \mu_{4}=-\frac{1}{2}$, $\cos \mu_{5}=-\frac{1}{2} \sqrt{3}$.
Upon calculating the $u_{k i}$ with this difference equation we have

\begin{tabular}{rrrrrrrrr}
$i$ & $=$ & 0 & 1 & 2 & 3 & 4 & 5 & 6 \\
\hline$u_{1 i}=$ & 0 & 1 & $\sqrt{3}$ & 2 & $\sqrt{3}$ & 1 & 0 \\
$u_{2 i}=$ & 0 & 1 & 1 & 0 & -1 & -1 & 0 \\
$u_{3 i}=$ & 0 & 1 & 0 & -1 & 0 & 1 & 0 \\
$u_{4 i}=$ & 0 & 1 & -1 & 0 & 1 & -1 & 0 \\
$u_{5 i}=$ & 0 & 1 & $-\sqrt{3}$ & 2 & $-\sqrt{3}$ & 1 & 0 \\
\hline
\end{tabular}

This particular example is remarkable in that the values obtained for the $\lambda_{k}$ and for the $u_{k i}$ not only are exact for the difference equation but are also exact for the differential system from which we started. We know, of course, that the correct $\lambda$ 's are given by

$$
\lambda_{k}=k \pi \text {. }
$$

We readily see that the values obtained numerically for $u_{k i}$ are also the values of the true characteristic functions

$$
\begin{aligned}
& u_{1}(x)=2 \sin \pi x, \\
& u_{2}(x)=\frac{2}{\sqrt{3}} \sin 2 \pi x, \\
& u_{3}(x)=\sin 3 \pi x, \\
& u_{4}(x)=\frac{2}{\sqrt{3}} \sin 4 \pi x, \\
& u_{5}(x)=2 \sin 5 \pi x .
\end{aligned}
$$

When $p(x)$ or $q(x)$ are different from zero, the results will no longer be exact but only approximate.

Several examples follow, showing the numerical calculations and comparing the values obtained with the correct values. Each of the examples illustrates a slightly different aspect of the process. Examples 1 and 2 differ only in the number of intervals employed. Example 3 illustrates the case of variable coefficients. In examples 1, 2, 3 the coefficients satisfy the conditions of continuity imposed in the foregoing discussion, whereas in 4 and 5 these conditions are not satisfied.

The values of the characteristic functions were also computed in the case of Example 4, and compared with the correct values. 
Characteristic numbers: Example 1

Differential equation: $\frac{d^{2} u}{d x^{2}}+2 \frac{d u}{d x}+\lambda^{2} u=0$

Boundary conditions: $u=0$ at $x=0$ and at $x=1$

Interval: $h=1 / 8$

Difference equation: $V_{i, i+1}=\frac{1}{8}\left[7 V_{i-1, j}+9 V_{i+1, i}\right]-V_{i, j-1}$

Boundary conditions: $V_{0, i}=V_{8, i}=0$

\begin{tabular}{|c|c|c|c|c|c|c|c|c|}
\hline 0 & 1 & 2 & 3 & 4 & 5 & 6 & 7 & 8 \\
\hline 0 & 2097152 & 0 & 0 & 0 & 0 & 0 & 0 & 0 \\
\hline 0 & 0 & 917504 & 0 & 0 & 0 & 0 & 0 & 0 \\
\hline 0 & -1064960 & 0 & 802816 & 0 & 0 & 0 & 0 & 0 \\
\hline 0 & 0 & -946176 & 0 & 702464 & 0 & 0 & 0 & 0 \\
\hline 0 & 512 & 0 & -840448 & 0 & 614656 & 0 & 0 & 0 \\
\hline 0 & 0 & 1120 & 0 & -746368 & 0 & 537824 & 0 & 0 \\
\hline 0 & 748 & 0 & 1764 & 0 & -662676 & 0 & 470596 & 0 \\
\hline 0 & 0 & 1519 & 0 & 2401 & 0 & -588245 & 0 & 0 \\
\hline
\end{tabular}

Characteristic equation:

$F(\mu)=\cos 7 \mu+(1.09375) \cos 5 \mu+(1.15869) \cos 3 \mu+(1.19191) \cos \mu=0$

\begin{tabular}{|c|c|c|c|c|}
\hline$k$ & $\mu_{k}$ & ${ }^{\mathrm{a}} \lambda_{k}^{*}$ & ${ }^{\mathrm{b}} \lambda_{k}$ & Relative error \\
\hline & Degrees & & & \\
\hline 1 & 23. 561 & 3. 2898 & 3. 2969 & -0.00215 \\
\hline 2 & 45. 448 & 6. 3457 & 6. 3623 & -0.00261 \\
\hline 3 & 67. 686 & 9. 4507 & 9. 4777 & -0.00285 \\
\hline 4 & 90 & 12. 5664 & 12. 6061 & -0.00315 \\
\hline 5 & 112. 314 & 15. 6820 & 15. 7398 & -0.00367 \\
\hline 6 & 134. 552 & 18. 7870 & 18. 8761 & -0.00472 \\
\hline 7 & 156. 439 & 21. 8430 & 22. 0139 & -0.00776 \\
\hline
\end{tabular}

a $\lambda_{k}^{*}=$ calculated value

$\mathrm{b}_{k}=$ correct value. 
Differential equation: $\frac{d^{2} u}{d x^{2}}+2 \frac{d u}{d x}+\lambda^{2} u=0$

Boundary conditions: $u=0$ at $x=0$ and at $x=1$

Interval: $h=1 / 16$

Difference equation: $V_{i, j+1}=\frac{1}{16}\left[15 V_{i-1, i}+17 V_{i+1, i}\right]-V_{i, j-1}$

Boundary conditions: $V_{o, i}=\mathrm{V}_{16, i}=0$

\begin{tabular}{|c|c|c|c|c|c|c|c|c|c|c|c|c|c|c|c|c|}
\hline 0 & 1 & 2 & 3 & 4 & 5 & 6 & 7 & 8 & 9 & 10 & 11 & 12 & 13 & 14 & 15 & 16 \\
\hline 0 & 115292 & 0 & 0 & 0 & 0 & 0 & 0 & 0 & 0 & 0 & 0 & 0 & 0 & 0 & 0 & 0 \\
\hline 0 & 0 & 54043 & 0 & 0 & 0 & G & 0 & 0 & 0 & 0 & 0 & 0 & 0 & 0 & 0 & 0 \\
\hline 0 & -57871 & 0 & 50665 & 0 & 0 & 0 & 0 & 0 & 0 & 0 & 0 & 0 & 0 & 0 & 0 & 0 \\
\hline 0 & 0 & -54465 & 0 & 47499 & 0 & 0 & 0 & 0 & 0 & 0 & 0 & 0 & 0 & 0 & 0 & 0 \\
\hline 0 & 1. 7592 & 0 & -51259 & 0 & 44530 & 0 & u & 0 & 0 & 0 & 0 & 0 & 0 & 0 & 0 & 0 \\
\hline 0 & 0 & 4. 1232 & 0 & -48241 & 0 & 41747 & 0 & 0 & 0 & 0 & 0 & 0 & 0 & 0 & 0 & 0 \\
\hline 0 & 2. 6216 & 0 & 6. 9578 & 0 & -45400 & 0 & 39138 & 0 & 0 & 0 & 0 & 0 & 0 & 0 & 0 & 0 \\
\hline 0 & 0 & 5. 7273 & 0 & 10. 147 & 0 & -42726 & 0 & 36692 & 0 & 0 & 0 & 0 & 0 & 0 & 0 & 0 \\
\hline 0 & 3. 4636 & 0 & 9. 1926 & 0 & 13.590 & 0 & -40208 & 0 & 34399 & 0 & 0 & 0 & 0 & 0 & 0 & 0 \\
\hline 0 & 0 & 7. 2869 & 0 & 12. 910 & 0 & 17. 199 & 0 & -37838 & 0 & 32249 & 0 & 0 & 0 & 0 & 0 & 0 \\
\hline 0 & 4. 2787 & 0 & 11. 356 & 0 & 16. 788 & 0 & 20.902 & 0 & -35608 & 0 & 30233 & 0 & 0 & 0 & c & 0 \\
\hline 0 & 0 & 8. 7900 & 0 & 15. 573 & 0 & 20. 748 & 0 & 24.634 & 0 & $-335 \mathrm{c} 8$ & 0 & 28344 & 0 & 0 & 0 & 0 \\
\hline 0 & 5. 0606 & 0 & 13. 431 & 0 & 19.856 & 0 & 24. 723 & 0 & 28.344 & 0 & -31532 & 0 & 26572 & 7 & 0 & 0 \\
\hline 0 & 0 & 10. 225 & 0 & 18. 116 & 0 & 24. 136 & 0 & 28.658 & 0 & 31.985 & 0 & -29672 & 0 & 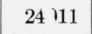 & 0 & 0 \\
\hline 0 & 5.8035 & 0 & 15.403 & 0 & 22.772 & 0 & 28.354 & 0 & 32.508 & 0 & 35.522 & 0 & -27921 & 0 & 23354 & 0 \\
\hline 0 & 0 & 11. 581 & 0 & 20.519 & 0 & 27.339 & 0 & 32.463 & 0 & 36. 233 & 0 & 38.924 & 0 & -26274 & 0 & 0 \\
\hline
\end{tabular}

Characteristic equation:

$F(\mu)=\cos 15 \mu+(1.05469) \cos 13 \mu+(1.10275) \cos 11 \mu+(1.14366) \cos 9 \mu$ $+(1.17695) \cos 7 \mu+(1.20225) \cos 5 \mu+(1.21928) \cos 3 \mu$

$+(1.22784) \cos \mu=0$.

\begin{tabular}{|c|c|c|c|c|}
\hline$k$ & $\mu_{k}$ & ${ }^{\mathrm{a}} \lambda_{k}^{*}$ & ${ }^{\mathrm{b}} \boldsymbol{\lambda}_{k}$ & Relative error \\
\hline & Degrees & & & \\
\hline 1 & 11. 800 & 3. 2952 & 3. 2969 & -0.00052 \\
\hline 2 & 22. 769 & 6. 3583 & 6. 3623 & -.00063 \\
\hline 3 & 33. 917 & 9. 4715 & 9. 4777 & -.00065 \\
\hline 4 & 45. 112 & 12. 5976 & 12. 6061 & -.00067 \\
\hline 5 & 56.325 & 15. 7289 & 15. 7398 & -.00069 \\
\hline 6 & 67.546 & 18. 8625 & 18. 8761 & -.00072 \\
\hline 7 & 78. 772 & 21. 9974 & 22. 0139 & -.00075 \\
\hline 8 & 90 & 25. 1327 & 25. 1526 & -.00079 \\
\hline 9 & 101. 228 & 28. 2681 & 28. 2920 & -.00084 \\
\hline 10 & 112. 454 & 31. 4030 & 31. 4318 & -.00092 \\
\hline 11 & 123. 675 & 34. 5366 & 34.5720 & -.00102 \\
\hline 12 & 134. 888 & 37. 6679 & 37. 7124 & -.00118 \\
\hline 13 & 146. 083 & 40. 7940 & 40. 8529 & -.00144 \\
\hline 14 & 157. 231 & 43. 9072 & 43. 9937 & -.00197 \\
\hline 15 & 168. 200 & 46. 9703 & 47. 1345 & -.00348 \\
\hline
\end{tabular}

$\Delta \lambda_{k}^{*}=$ calculated value

$\mathrm{b} \lambda_{k}=$ correct value 
Characteristic numbers: Example 3

Differential equation: $4 x \frac{d^{2} u}{d x^{2}}+\lambda^{2} u=0$

Boundary conditions: $u=0$ at $x=1$ and at $x=4$

Change variable: $x=s^{2}$

Normal equation: $\frac{d^{2} u}{d s^{2}}-\frac{1}{s} \frac{d u}{d s}+\lambda^{2} u=0, u=0$ at $s=1$ and at $s=2$

Interval: $h=1 / 8$

Difference equation: $V_{i, i+1}=\frac{1}{2 i}\left[(2 i+1) V_{i-1, j}+(2 i-1) V_{i+1, i}\right]-V_{i, i-1}$

Boundary conditions: $V_{8, i}=V_{10, j}=0$

\begin{tabular}{|c|c|c|c|c|c|c|c|c|}
\hline 8 & 9 & 10 & 11 & 12 & 13 & 14 & 15 & 16 \\
\hline 0 & 13178880 & 0 & 0 & 0 & 0 & 0 & 0 & 0 \\
\hline 0 & 0 & 6918912 & 0 & 0 & 0 & 0 & 0 & 0 \\
\hline 0 & -6644352 & 0 & 7233408 & 0 & 0 & 0 & 0 & 0 \\
\hline 0 & 0 & -7023744 & 0 & 7534800 & 0 & 0 & 0 & 0 \\
\hline 0 & 10816 & 0 & -7384104 & 0 & 7824600 & 0 & 0 & 0 \\
\hline 0 & 0 & 20202 & 0 & -7728000 & 0 & 8104050 & 0 & 0 \\
\hline 0 & 8263.667 & 0 & 28497 & 0 & -8057475 & 0 & 8374185 & 0 \\
\hline 0 & 0 & 15547 & 0 & 35937. 5 & 0 & -8374185 & 0 & 0 \\
\hline
\end{tabular}

Characteristic equation:

$F(\mu)=\cos 7 \mu+(1.03333) \cos 5 \mu+(1.05506) \cos 3 \mu+(1.06578) \cos \mu=0$.

\begin{tabular}{|c|c|c|c|c|}
\hline$k$ & $\mu_{k}$ & ${ }^{\mathrm{a}} \lambda_{k}^{*}$ & ${ }^{\mathrm{b}} \lambda_{k}$ & Relative error \\
\hline & Degrees & & & \\
\hline 1 & 22. 873 & 3. 1936 & 3. 1966 & -0.00094 \\
\hline 2 & 45. 164 & 6. 3060 & 6. 3124 & -0.00101 \\
\hline 3 & 67.568 & 9. 4343 & 9.4445 & -0.00108 \\
\hline 4 & 90 & 12. 5664 & 12. 5812 & -0.00118 \\
\hline 5 & 112. 432 & 15. 6984 & 15. 7199 & -0.00137 \\
\hline 6 & 134. 836 & 18. 8267 & 18. 8595 & -0.00174 \\
\hline 7 & 157. 127 & 21. 9391 & 21. 9997 & -0.00275 \\
\hline
\end{tabular}

a $\lambda_{k}^{*}=$ calculated value.

$\mathrm{b}_{\lambda_{k}}=$ correct value 
Characteristic numbers: Example 4

(Differential equation with singular point)

Differential equation: $\frac{d^{2} u}{d x^{2}}+\frac{1}{x} \frac{d u}{d x}+\lambda^{2} u=0$

Boundary conditions: $u$ finite at $x=0, u=0$ at $x=1$

Interval: $h=1 / 8$
Difference equation: $V_{i, j+1}=\frac{1}{2 i}\left[(2 i-1) V_{i-1, i}\right.$

$$
\left.+(2 i+1) V_{i+1, i}\right]=V_{i, i-1}
$$

Boundary conditions: $V_{0, j+1}=4 V_{1, i}-2 V_{0, i}-V_{0, i-1}$ $V_{8, j}=0$

\begin{tabular}{|c|c|c|c|c|c|c|c|c|}
\hline 0 & 1 & 2 & 3 & 4 & 5 & 6 & 7 & 8 \\
\hline 1 & 0 & 0 & 0 & 0 & 0 & 0 & 0 & 0 \\
\hline-1 & 0. 25 & 0 & 0 & 0 & 0 & 0 & 0 & 0 \\
\hline 2 & -0.5 & 0. 1875 & 0 & 0 & 0 & 0 & 0 & 0 \\
\hline-5 & 1. 03125 & -0.375 & 0. 15625 & 0 & 0 & 0 & 0 & 0 \\
\hline 12. 125 & -2.5625 & 0. 78125 & -0.3125 & 0. 13672 & 0 & 0 & 0 & 0 \\
\hline-29.5 & 6. 203125 & -1.9375 & 0. 65430 & -0.27344 & 0. 12305 & 0 & 0 & 0 \\
\hline 71. 6875 & -15.09375 & 4. 68896 & -1.62109 & 0.57422 & -0.24609 & 0. 11279 & 0 & 0 \\
\hline-174.25 & 36. 67407 & -11.40918 & 3. 92310 & -1.421875 & 0. 51782 & -0.22558 & 0. 10473 & 0 \\
\hline 423. 50879 & -89.14502 & 27. 72046 & -9.54541 & 3. 44104 & -1.28174 & 0. 47534 & -0.20947 & 0 \\
\hline
\end{tabular}

Characteristic equation:

$F(\mu)==\cos 8 \mu+2 \cos 7 \mu+(-0.21438) \cos 6 \mu+(1.57126) \cos 5 \mu+(-0.32580) \cos 4 \mu+(1.41947) \cos 3 \mu$ $\dashv(-0.35057) \cos 2 \mu+(1.36154) \cos \mu+(-0.17790)=0$

\begin{tabular}{|c|c|c|c|c|}
\hline$k$ & $\mu_{k}$ & ${ }^{\mathrm{a}} \lambda_{k}^{*}$ & ${ }^{\mathrm{b}} \lambda_{k}$ & Relative error \\
\hline & Degrees & & & \\
\hline 1 & 17. 212 & 2. 4032 & 2. 4048 & -0.00067 \\
\hline 2 & 39. 518 & 5. 5178 & 5. 5201 & -0.00042 \\
\hline 3 & 62. 000 & 8. 6568 & 8. 6537 & +0.00036 \\
\hline 4 & 84. 623 & 11. 8156 & 11. 7915 & 0. 00204 \\
\hline 5 & 107. 545 & 15. 0161 & 14. 9309 & 0.00571 \\
\hline 6 & 131. 028 & 18. 2950 & 18. 0711 & 0.01239 \\
\hline 7 & 155. 614 & 21. 7278 & 21. 2116 & 0.02434 \\
\hline
\end{tabular}

จ $\lambda_{k}^{*}=$ calculated value.

$\mathrm{b} \lambda_{\mathrm{c}}=$ correct value. 
Calculation of characteristic functions for example 4

$$
\begin{array}{lr}
V_{k, i-1}=\frac{1}{2 i-1}\left\{\left[4 i \cos \mu_{k}\right] V_{k i}-(2 i+1) V_{k, i+1}\right\} \\
V_{k 8}=0, V_{k 7}=1 & h=1 / 8
\end{array}
$$

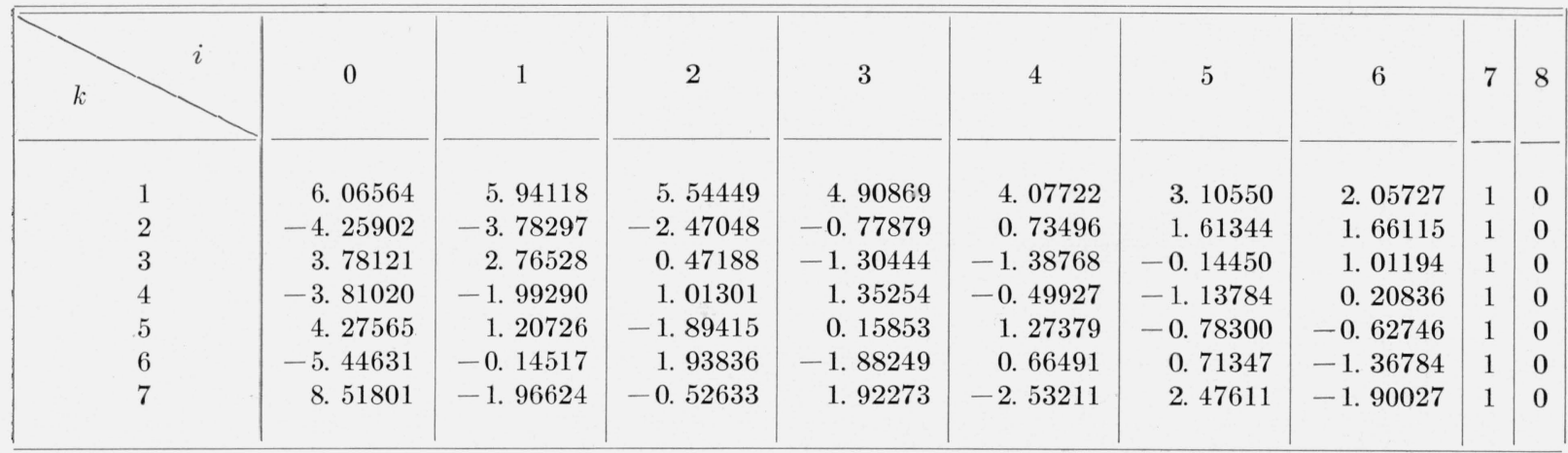

\begin{tabular}{|c|c|c|c|c|c|c|c|c|c|}
\hline$k$ & 0 & 1 & 2 & 3 & 4 & 5 & 6 & 7 & 8 \\
\hline 1 & 1 & 0. 97948 & 0. 91408 & 0. 80926 & 0. 67218 & 0. 51198 & 0. 33917 & 0. 16486 & 0 \\
\hline 2 & 1 & 0.88823 & 0.58006 & 0. 18286 & -0.17257 & -0.37883 & -0.39003 & -0.23480 & 0 \\
\hline 3 & 1 & 0. 73132 & 0. 12480 & -0.34498 & -0.36699 & -0.03822 & 0. 26762 & 0. 26447 & 0 \\
\hline 4 & 1 & 0. 52304 & -0.26587 & -0.35498 & 0. 13104 & 0. 29863 & -0.05468 & -0.26245 & 0 \\
\hline 5 & 1 & 0. 28236 & -0.44301 & 0. 03708 & 0. 29792 & -0.18313 & -0.14675 & 0. 23388 & 0 \\
\hline 6 & 1 & 0. 02665 & -0.35590 & 0. 34565 & -0.12208 & -0.13100 & 0. 25115 & -0.18361 & 0 \\
\hline 7 & 1 & -0.23083 & -0.06179 & 0. 22573 & -0.29727 & 0. 29069 & -0.22309 & 0. 11740 & 0 \\
\hline
\end{tabular}

Normalized so that $V_{k, 0}=1$

\begin{tabular}{|c|c|c|c|c|c|c|c|c|c|}
\hline$k$ & 0 & 1 & 2 & 3 & 4 & 5 & 6 & 7 & 8 \\
\hline 1 & 1 & 0. 97754 & 0. 91166 & 0. 80679 & 0. 66993 & 0. 51014 & 0. 33788 & 0. 16421 & 0 \\
\hline 2 & 1 & 0. 88447 & 0.57765 & 0. 18368 & -0.16840 & -0.37274 & -0.38424 & -0.23137 & 0 \\
\hline 3 & 1 & 0.72818 & 0. 13078 & -0.33158 & -0.35628 & -0.03825 & 0. 25859 & 0. 25597 & 0 \\
\hline 4 & 1 & 0.52632 & -0. 24188 & -0.33776 & 0. 12078 & 0. 28180 & -0.05069 & -0.24713 & 0 \\
\hline 5 & 1 & 0. 30138 & -0.40077 & 0. 02667 & 0. 27086 & -0.16394 & -0.13336 & 0. 21102 & 0 \\
\hline 6 & 1 & 0. 07789 & -0.31640 & 0. 29149 & -0.09897 & -0.11348 & 0. 21266 & -0.15483 & 0 \\
\hline 7 & 1 & -0.12066 & -0.07480 & 0. 18221 & -0.22704 & 0. 21788 & -0.16591 & 0. 08703 & 0 \\
\hline
\end{tabular}

True values: $J_{0}\left(j_{0 k} x\right)$ 
Characteristic numbers: Example 5

Partial differential equation: $\frac{1}{x} \frac{\partial^{2} u}{\partial t^{2}}=\frac{\partial}{\partial x}\left(\frac{1}{x} \frac{\partial u}{\partial x}\right)$

Boundary conditions: $u$ finite at $x=0, u=0$ at $x=1$

Ordinary differential equation: $\frac{d^{2} u}{d x^{2}}-\frac{1}{x} \frac{d u}{d x}+\lambda^{2} u=0$

Difference equation: $V_{i, j+1}=\frac{1}{2 i}\left[(2 i+1) V_{i-1, i}+(2 i-1) V_{i+1, i}\right]-V_{i, i-1}$

Boundary conditions: $V_{0, i}=V_{8, i}=0$

\begin{tabular}{|c|c|c|c|c|c|c|c|c|}
\hline \hline 0 & 1 & 2 & 3 & 4 & 5 & 6 & 7 & 8 \\
\cline { 1 - 7 } 0 & 40320 & 0 & 0 & 0 & 0 & 0 & 0 \\
0 & 0 & 25200 & 0 & 0 & 0 & 0 & 0 & 0 \\
0 & -27720 & 0 & 29400 & 0 & 0 & 0 & 0 & 0 \\
0 & 0 & -37800 & 0 & 33075 & 0 & 0 & 0 & 0 \\
0 & 8820 & 0 & -45937.5 & 0 & 36382.5 & 0 & 0 & 0 \\
0 & 0 & 14371.875 & 0 & -52820 & 0 & 39414.375 & 0 & 0 \\
0 & -1634.0625 & 0 & 18604.6875 & 0 & -59121.5625 & 0 & 42229.6875 & 0 \\
0 & 0 & -2460.9375 & 0 & 22118.90625 & 0 & -64752.1875 & 0 & 0 \\
\hline
\end{tabular}

Characteristic equation:

$F(\mu)=\cos 7 \mu+(1.64286) \cos 5 \mu+(1.95982) \cos 3 \mu+(2.10044) \cos \mu=0$

\begin{tabular}{|c|c|c|c|c|}
\hline$k$ & $\mu_{k}$ & ${ }^{\mathrm{a}} \lambda_{k}^{*}$ & ${ }^{\mathrm{b}} \lambda_{k}$ & Relative error \\
\hline & Degrees & & & \\
\hline 1 & 27. 145 & 3. 7901 & 3. 8318 & -0.0109 \\
\hline 2 & 49. 044 & 6. 8479 & 7. 0155 & -.0239 \\
\hline 3 & 69. 928 & 9. 7638 & 10. 1735 & -.0403 \\
\hline 4 & 90 & 12. 5664 & 13. 3237 & -.0568 \\
\hline 5 & 110. 072 & 15. 3690 & 16. 4706 & -.0669 \\
\hline 6 & 130. 956 & 18. 2849 & 19. 6158 & -.0678 \\
\hline 7 & 152.855 & 21. 3427 & 22. 7601 & -.0623 \\
\hline
\end{tabular}

$8 \lambda_{k}^{*}=$ calculated value.

$\mathrm{b}_{\lambda_{k}}=$ correct value.

Los Angeles, June 20, 1949. 